\title{
Anti-ISI Demodulation Scheme and Its Experiment-based Evaluation for Diffusion-based Molecular Communication
}

\author{
Haoyang Zhai, Qiang Liu*, Member, IEEE, Athanasios V. Vasilakos, and Kun Yang, Senior Member, IEEE
}

\begin{abstract}
In diffusion-based molecular communication (MC), the most common modulation technique is based on the concentration of information molecules. However, the random delay of molecules due to the channel with memory causes severe inter-symbol interference (ISI) among consecutive signals. In this paper, we propose a detection technique for demodulating signals, the increase detection algorithm (IDA), to improve the reliability of concentration-encoded diffusion-based molecular communication. The proposed IDA detects an increase (i.e., a relative concentration value) in molecule concentration to extract the information instead of detecting an absolute concentration value. To validate the availability of IDA, we establish a real physical tabletop testbed. And we evaluate the proposed demodulation technique using bit error rate (BER) and demonstrate by the tabletop molecular communication platform that the proposed IDA successfully minimizes and even isolates ISI so that a lower BER is achieved than the common demodulation technique.
\end{abstract}

Index Terms-Molecular communication, concentration-based encoding, diffusion channel, increase detection algorithm, demodulation technique.

\section{INTRODUCTION}

$\mathbf{I}$ $\mathrm{N}$ molecular communication (MC) [1]-[3], capable of communication at the nanoscale, using biological nanomachines, can unlock new areas of engineering. Inspired by how biological cells communicate, MC uses chemical signals as carriers of information instead of electromagnetic waves. Bio-nanomachines can communicate with each other from nanoscale to microscale environments. Transmitters encode information into molecules and transmit the informationencoded molecules in the environment. Encoded molecules then propagate in various media, such as diffusion channel, neural channel etc. Receiver bio-nanomachines finally detect the propagating molecules and extract the information carried by molecules.

Propagation methods of molecules include active schemes, which need the energy to direct the information molecules

This work is supported by the National Natural Science Foundation of China under Grant No.61471102 and the Fundamental Research Funds for the Central Universities under Grant No.ZYGX2015J001.

Haoyang Zhai and Qiang Liu are with the School of Communication Information Engineering, University of Electronic Science and Technology of China (e-mails: hyzhai@std.uestc.edu.cn; liuqiang@uestc.edu.cn). Asterisk indicates corresponding author.

Kun Yang is with the School of Computer Science Electronic Engineering, University of Essex, U.K. and also with the School of Communication Information Engineering, University of Electronic Science and Technology of China (e-mail: kunyang@essex.ac.uk).

Athanasios V. Vasilakos is with Lulea University of Technology, Sweden (e-mail: vasilako@ath.forthner.gr). from the transmitter towards the receiver, and passive schemes, where the transmitter has no direct influence on the path taken by the emitted molecules. Passive schemes, such as free diffusion, are simpler to implement and more easily enable the formation of ad hoc networks between bio-nanomachines.

It is common to find diffusion-based communication in cellular systems, when small molecules need to quickly travel short distances [4]. Because of its simplicity and its current implementation in nature, diffusion has also often been chosen by communication researchers for the design of synthetic nanonetworks. There are a number of characteristics that distinguish the diffusive channel from conventional communication channels. For example, it features randomly-moving molecules instead of propagating electromagnetic radiation. So the propagation time critically depends on the distance between the transmitter and receiver, which leads to severe ISI due to the lingering presence of emitted molecules.

There are two significant open problems in current studies of MC:(1) to determine and characterize the signal strength of the molecular propagation channel, and (2) to find efficient modulation schemes such that receiver bio-nanomachines can identify the information conveyed by the chemical molecules as accurate as possible. In this case, "signal strength" can be easily understood as the molecule concentration or amplitude. Networks of bio-nanomachines that communicate through MC are expected to be biologically friendly and operable in or with biological systems. Applications of such networks are anticipated to develop future health monitoring and drug delivery systems.

Demodulation is a basic technique in the signaling sublayer in the layered architecture of MC [5]. In conventional concentration-encoded MC system, receiver bio-nanomachines depend on a fixed threshold to extract the information from the molecule concentration. Namely, if the molecule concentration detected by the receiver is above the threshold, receiver bionanomachines will demodulate the signal as an information bit "1"; otherwise, they will detect it as an information bit " 0 ". The traditional demodulation techniques which demodulate the signal as " 1 " or " 0 " only depending on a threshold of the received molecule concentration are ineffective in the presence of ISI and other types of noise, which result from the stochastic property of Brownian motion, the imperfection of bio-nanomachines, the chemical reactions of molecules and other factors.

The motivation of this paper is therefore to design a robust demodulation technique that can avoid the impact of ISI 
and other types of noise in concentration-encoded diffusionbased MC. Toward this end, this paper proposes the increase detection algorithm (IDA) for concentration-encoded MC, and evaluates its performance in terms of BER under different parameters using a physical tabletop testbed. Experimental results show that the IDA can significantly mitigate the ISI and reduce the effect of noise in concentration-encoded molecular communication.

The contributions of this paper are listed as the following. First, we propose a novel demodulation technique, the IDA, which was first presented in [6]. In [6], some simulation experiments were carried out considering the performance of bit error rate (BER) with the signal to noise ratio (SNR), while a physical tabletop testbed is established to evaluate the availability of IDA in this paper. However, the power of noise is difficult to quantify with the inexpensive and simple platform, so we evaluate the BER performance with other important parameters, such as the length of each time slot and so on. With IDA, the inter-symbol interference (ISI), the most common interference in diffusion-based MC, can be avoided primarily. Second, we establish a real physical tabletop testbed to validate the proposed demodulation algorithm and achieve the performance of IDA using the indicator of BER through a series of experiments. Further, the relationships between BER and other important parameters are presented in this paper. To the best of our knowledge, it is the first time to propose a demodulation technique that can significantly mitigate the impact of ISI and evaluate its performance through a physical tabletop testbed in concentration-encoded diffusion-based MC.

This paper is organized as follows. Section II presents the current research efforts in this field of MC. In Section III, we introduce the basic system model of concentration-encoded diffusion-based molecular communication. In Section IV, the IDA is represented, analysed theoretically and discussed in detail. A physical tabletop testbed and the experimental setup are introduced in Section V. In section VI, we validate the IDA with the hardware platform and evaluate the BER performance of the proposed IDA comparing with the traditional demodulation algorithm. Finally, we conclude this paper in Section VII.

\section{RELATED WORK}

There are many articles investigating the communication mechanism of molecular communication (MC) based on different information carriers or propagation media. Some typical cases are like diffusion-based MC [7], neural communication [8], motor-based MC [9], blood vessel MC [10], and bacteriabased MC [11].

Concentration-encoded molecular communication (CEMC) [12] uses a single type of molecule to transmit information. The transmitter modulates the amplitude of the transmitting rate of molecules, and correspondingly, the receiver decodes the information symbols by strength-based [13] or samplingbased [14] detection schemes. Hence the detection schemes in CEMC are also known as threshold-based detection. The intensity and the strength of signal respectively mean the instantaneous amplitude of concentration of molecules at any time instant and the total number of accumulated molecules in the entire symbol duration. In this paper, the concentration of molecules is explained in terms of the number of molecules per unit sensing volume of the receiver.

Some existing literature aims to improve the reliability performance of the molecular communication system, and the detection and demodulation method at the receiver is an important part. In [15], receivers are designed with different signal estimation technologies to eliminate the impact of ISI and noise. [16] designs an optimal detector for the MC channels with enzymes that help reduce the ISI. Further, in [17], a detection technique at receiver is developed based on matrix operations or likelihood calculations. Based on the common demodulation method using a fixed threshold, [18] proposes a low-complexity detection algorithm named the adaptive threshold detection to resolve the problem caused by ISI in diffusion-based MC. Similarly, [19] proposes an adaptive threshold variation (ATV) algorithm, which is the latest ISI mitigation method based on an adaptive threshold and its BER performance is best at present. However, the transmitting quantity of molecules (i.e., 500) and the step size adjusting the threshold (i.e., 1) in ATV algorithm are too small to match the real situation. Futhermore, various strategies from conventional communications have also been proposed to improve communication via diffusion, including channel coding in [20], emitting Gaussian pulses in [21], network coding in [22], and a forward error correction coding over $\mathrm{MC}$ is proposed to achieve better reliability performance in [23]. However, the feasibility of such strategies is unknown. The ISI is always present in these MC systems and remains to be addressed.

Unlike the aforementioned ISI mitigation techniques, the proposed IDA uses a dynamic characteristic of molecule concentration in diffusion-based molecular communication. Similarly, [24] investigates the impact of two different receiver reaction mechanisms on the performance of molecular communication networks and derive analytical expressions for the mean and variance of the output signals. Focusing on improving the reliability of each reception, the research in [24] does not consider the effect of ISI. Another similar work is introduced in [25], where the proposed modulation algorithm relies on the dynamic properties of oscillating and propagating patterns in molecule concentration, such as the dynamic amplitude and period. While the technique introduced in [25] is a kind of modulation methods, the proposed IDA is a demodulation algorithm and is more generic in concentrationencoded MC. Furthermore, the IDA proposed in this paper detects an increase in molecule concentration to extract the information encoded in molecules.

Since it's difficult to manufacture nanoscale devices at present, the first tabletop molecular communication testbed capable of transmitting a text message across the room using chemical signaling, which was presented in [26], is a very powerful tool to facilitate the theoretical research, and provide some insights on manufacturing and implementation at nanoscale. The platform was purposefully designed to be inexpensive and simple, so we develop our experimental tool following this platform, which will be introduced in Section 


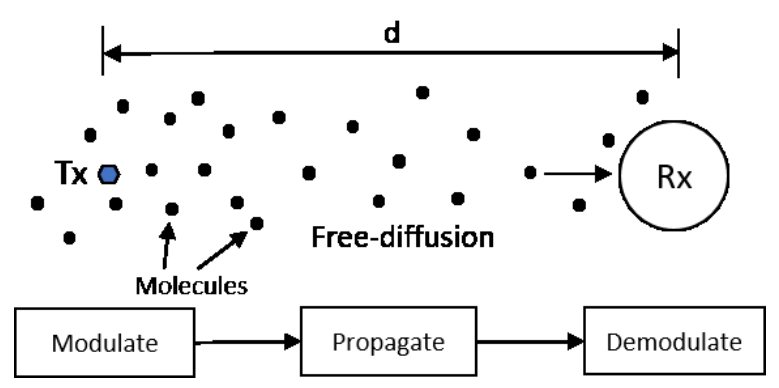

Fig. 1. The communication process in diffusion-based molecular communication system. Tx indicates transmitter; $\mathrm{Rx}$ is receiver.

V. In [27], theoretical models are modified to create more realistic channel models based on experimental observations using a tabletop molecular communication platform, while [28] introduces corrections to the previous theoretical models of the end-to-end system impulse response based on the observed data from experimentation. [29] proposes an endto-end channel model for molecular communication systems with metal-oxide sensors, which focuses on the recently developed tabletop molecular communication platform in particular. Moreover, [30] presents a molecular multiple-input multiple-output (MIMO) communication link to deliver two data streams in a spatial domain, while [31] shows a molecular MIMO communication link with drift and an artificial flow exists in the environment.

To the best of our knowledge, it is the first time to propose an anti-ISI demodulation algorithm with low complexity and evaluate its performance in detail by a tabletop molecular communication platform. Unlike any threshold-based detection schemes, we adopt a completely different approach to detect received information in this paper, which uses the relative concentration detection (i.e., the difference value of two adjacent absolute values) to improve communicating reliability. And we evaluate the performance of the IDA proposed in this paper by the macroscopic molecular communication platform, which will be developed in Sections V and VI.

\section{SYSTEM MODEL}

We consider the diffusion-based MC system illustrated in Fig. 1. It is composed of a transmitter (Tx), a receiver $(\mathrm{Rx})$ and the diffusion channel (i.e., the environment) in which molecules propagate. The distance between $\mathrm{Tx}$ and $\mathrm{Rx}$ is given by $d$.

We also consider a time-slotted MC system with a fixed time slot length of $T$ where one bit is transmitted. For simplicity, Tx and Rx are assumed to be perfectly synchronized, meaning that the time slots of $\mathrm{Tx}$ and $\mathrm{Rx}$ are aligned. To transmit a binary sequence $b[i]$, Tx emits a certain number of molecules or not into the environment at the beginning of each time slot. Molecules emitted by Tx propagate in the diffusion channel to reach $\mathrm{Rx}$ within the same time slot where the molecules are emitted or in a different time slot, owing to the probabilistic nature of the diffusion channel. Rx receives a binary sequence $\hat{b}[i]$ by using the demodulation technique presented in Section IV.
In our model, we consider a realistic type of macroscopic receiver, such as the receiver in [26], which can probably be disturbed by the accumulation of molecules around itself. In other words, the molecules reaching the receiver can not disappear immediately, but continue for a random period of time. Therefore, the MC system considered in this paper may suffer from ISI following the communication between Tx and Rx because the diffusion channel has a memory, which means that the signal of current slot is affected by all previous slot signals. In addition to ISI, diffusion noise (thermal noise) and other noises exist in the system. The ISI and noises degrade the communication performance, thus the MC system needs to be designed so that the impact of ISI and noises can be mitigated significantly.

\section{A. Modulation}

In molecular communication, information is modulated into various properties of molecules which $\mathrm{Tx}$ transmits or the manner that Tx transmits molecules, which is called the modulation technique. For instance, information is modulated into the number of molecules (i.e., concentration), the rate of molecule concentration changing (i.e., frequency), release time (i.e., phase), and type or structure of molecules, to form a signal. Different modulation techniques have been proposed for diffusion-based molecular communication (DMC). In [32], the researchers investigate a new energy model to understand how much energy is required to transmit messenger molecules in concentration shift keying (CSK) and [33] introduces a concentration-based and molecular-type-based modulation technique, which is called molecular shift keying (MoSK). The researchers also compare the achievable rate using a simple binary symmetric channel model. However, both CSK and MoSK suffer from ISI caused by molecules from previous transmissions. Molecule concentration shift keying (MCSK) proposed in [34] borrows the ideas from CSK and MoSK. In [35], the authors propose three novel modulation techniques, i.e., concentration-based, molecular-type-based, and molecular-ratio-based, using isomers as messenger molecules for nano communication networks via diffusion. Through multiple molecule types or isomers, MoSK, MCSK and other modulation techniques based on isomers are more resistant to interference than CSK, but they require complex molecular mechanisms at both the transmitter and the receiver for message synthesis and decoding.

Given that bio-nanomachines are small, limited in computational power and made of bio-materials, simple and biologically implementable modulation techniques are needed for MC. Concentration shift keying (CSK) is adopted in our system model because it is one of the simplest and easiest modulation techniques to implement on bio-nanomachines. CSK is similar with the amplitude shift keying (ASK) used in the classical communication. In this scheme, symbols are encoded in the number of molecules that the transmitter diffuse per time slot, which is called diffusion rate or transmission power. To represent $b$ bits, $2^{b}$ different molecule diffusion rates are utilized. In other words, different levels of molecule concentration are used to represent information bits in CSK. 
For example, if the carrier wave is a single point pulse, we use the presence or absence of a carrier wave to indicate an information bit " 1 " or " 0 ", which is the simplest on-off-keying (OOK) modulation as follows:

$$
N_{T x}(i)=\left\{\begin{aligned}
M, & \text { if } b[i]=1 \\
0, & \text { if } b[i]=0
\end{aligned}\right.
$$

where $N_{T x}(i)$ is the number of molecules that Tx emits at the beginning of the $i^{t h}$ slot. To modulate the information bit " 1 ", Tx emits $M$ message molecules into the environment, while no molecules are released to modulate the information bit " 0 ".

\section{B. Propagation}

The particles or messenger molecules released from the transmitter bio-nanomachines spread out through the medium by Brownian motion [18], [36]. In the 3-dimensional environment, a molecule emitted by $\mathrm{Tx}$ at time $t=0$ exists at time $t$ at the position with distance $x$, with the following probability:

$$
g(x, t)=\frac{1}{(4 \pi D t)^{3 / 2}} \exp \left(-\frac{x^{2}}{4 D t}\right) .
$$

$g(x, t)$ can also be regarded as the position distribution of a single molecule, where $D$ is the diffusion coefficient of the information molecules. In this paper, we assume that the diffusion coefficient $D$ is a constant, whose specific value has no direct effect on the result of experiments if $D$ remains the same.

\section{Demodulation}

After Tx emitting information molecules, the molecule concentration $C(x, t)$ at location $x$ and at time $t$ changes in reference to time and space as follows:

$$
C(x, t)=\int_{0}^{t} \frac{Q(\tau)}{(4 \pi D \tau)^{3 / 2}} \exp \left(-\frac{x^{2}}{4 D \tau}\right) d \tau
$$

where $Q(t)$ is the emission rate, $\tau$ is the time variable of integration, and $D$ is the diffusion constant of the molecule emitted by Tx. In this paper, $x$ is given in $\mu \mathrm{m}, Q(t)$ is in the number of molecules/second, and $t$ is in seconds. Here, $C(x, t)$ is given in the number of molecules $/ \mu \mathrm{m}^{3}$, indicating the "signal intensity" observed at location $x$.

The molecule concentration that Rx observes, namely, C(x, t) with $x=d$, is considered the intensity of the signal that $\mathrm{Rx}$ receives. If Tx emits $M$ molecules at time $t=0$, the signal intensity at $\mathrm{Rx}$, due to the $M$ molecules, denoted as $C(t)$, is represented as follows:

$$
C(t)=\frac{M}{(4 \pi D t)^{3 / 2}} \exp \left(-\frac{d^{2}}{4 D t}\right) .
$$

Let $V_{R}$ be the signal detection space of Rx. In the absence of noise, we calculate the signal that $\mathrm{Rx}$ receives in a time slot $i$ as,

$$
\operatorname{Sig}_{R x}=\int_{i T}^{(i+1) T} C(t) * V_{R} d t
$$

As for noise in theory, we can assume that the noise is zeromean additive white Gaussian noise (AWGN), which has been demonstrated in [28] and expressed as:

$$
\text { Noise } \sim \operatorname{Normal}\left(0, \sigma^{2}\right) \text {. }
$$

where $\sigma^{2}$ is the variance and indicates the noise strength. However, the noise in the environment is not the only additive white Gaussian noise but also other types of noise, such as the counting noise due to the memory of the propagation channel, and the noises caused by the chemical reactions of molecules, the imperfection of nano-devices and other factors. Moreover, the power of these noises is difficult to quantify in the experiments with the inexpensive and simple platform, which means that it is hard to calculate the specific value of the noise power. In this paper, we unify the noises as $S i g_{n o i s e}$, which contains all the noises at Rx. Therefore, the total signal at Rx is the sum of $S i g_{R x}$ and $S i g_{\text {noise: }}$ :

$$
S i g_{\text {total }}=S i g_{R x}+S i g_{n o i s e}
$$

which is represented by the sensor voltage in following experiments.

In contrast to the common demodulation techniques using a fixed threshold in molecule concentration, we propose a novel demodulation algorithm on the basis of the rate of change in molecule concentration in this paper. The proposed demodulation technique effectively detects an increase in the molecule concentration which always occurs at the beginning of each time slot or not. This technique, referred to as IDA, can reduce the influence of diffusion noise in the diffusion channel and ISI due to the accumulation of molecules in the environment. As a result, the proposed IDA can improve the reliability of this molecular communication system. We then present the detailed IDA in Section IV.

\section{InCRease Detection Algorithm}

In this section, we first illustrate how the IDA works in detail and its advantages compared with the traditional demodulation techniques based on a fixed threshold of molecule concentration. Finally, we discuss the complexity of the IDA.

In traditional demodulation techniques, $\mathrm{Rx}$ depends on a fixed threshold of molecule concentration to extract the information encoded in molecules. Namely, Rx compares the detected molecule concentration against the fixed threshold. The signal will be demodulated as a bit " 1 " if the molecule concentration obtained by $\mathrm{Rx}$ is above the threshold; otherwise, it will be received as a bit " 0 ". In this paper, we set the fixed threshold as the half of maximum molecule concentration at Rx (i.e., $C \_\max / 2$ ).

The common demodulation techniques based on a fixed concentration threshold is error-prone in diffusion-based MC. In diffusion-based MC, molecules emitted by Tx accumulate in the environment due to the slow and random natures of Brownian motion. This increases the chance of ISI and leads to high BER in demodulation. One can reduce the BER resulting from ISI by increasing the time slot length, which however decreases the information rate. This is the motivation for designing the IDA. The IDA aims to improve BER performance 


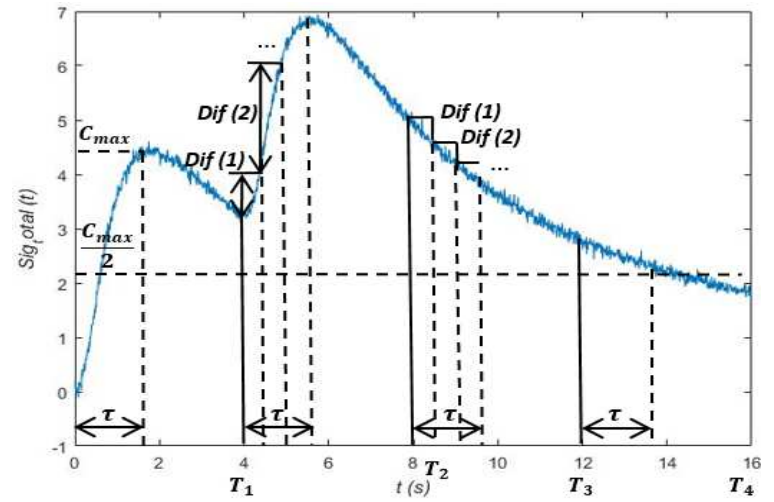

Fig. 2. The sampling process of the IDA in theory. Bit_rx $=$ "1100"

by detecting an increase in molecule concentration. In the IDA, if $S i g_{\text {total }}$ during the sampling time of each time slot has a noticeable rise, then the signal is demodulated as a bit " 1 ". Otherwise, the signal is demodulated as a bit "0". As shown in Fig. 2, we start to sample some molecule concentration values during an interval of $\tau$ from the beginning of each time slot $T$, which also means that we can eliminate the influence coming from the latter part of the curve presenting the molecule concentration changing. Then we calculate the difference value of two adjacent samples to detect the increase in the molecule concentration for several times, which can avoid the impact of slight fluctuations in the curve. It indicates a rise if the difference value is bigger than 0 . Otherwise, it means a descent or no change. Considering the effect of noise, we calculate multiple difference values during the interval of $\tau$ to identify the concentration variation trend.

In Figure 2, $\tau$ is the time interval from the start of each time slot to the peak of molecule concentration. In order to implement the IDA, we need first to calculate the value of $\tau$. Let the partial derivative of $C(t)$ of time $t$ equal to zero, which is given by (4). We can get the maximum value of $C(t)$ during a time slot at time $t_{0}$ when the molecule concentration reaches its peak value, which is given by (9).

From

$$
\frac{\partial C(t)}{\partial t}=0
$$

we have

$$
\begin{aligned}
t_{0} & =\frac{d^{2}}{6 D}, \\
C_{\max } & =C\left(t_{0}\right)=\frac{M}{\left(\frac{2}{3} \pi d^{2}\right)^{3 / 2}} e^{-\frac{3}{2}} .
\end{aligned}
$$

Note that, as shown in (10), $C_{\max }$ is determined by $d$ and $M$. In this paper, we use $\tau$ as the time period of sampling in each time slot:

$$
\tau=t_{0}=\frac{d^{2}}{6 D}
$$

Namely, $\tau$ is determined by $D$ and $d$, which is the time required for the molecule concentration to increase from the initial value to its peak value of $S_{i g_{\text {total }} \text {. }}$

The IDA is shown in Table I, where some parameters need to be pre-determined by using different techniques or
TABLE I

INCREASE DETECTION ALGORITHM

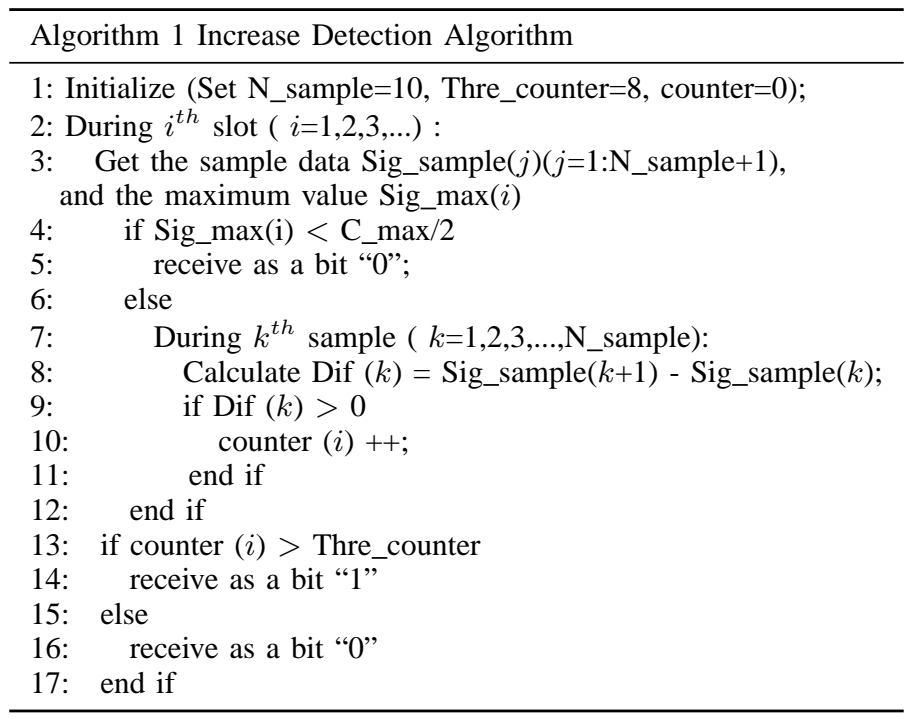

measurements, such as the Tx-Rx distance $(d)$, the time slot length $(T)$, the number of samplings ( $N \_$sample), and the threshold of the counter (Thre_counter). During the sampling time, if the maximum value of $S i g_{\text {total }}$ is smaller than $C_{\max } / 2$, the signal is demodulated as a bit " 0 ". Otherwise, we calculate the difference value of two adjacent samples at the beginning of each time slot, whose result is Dif(1) in the figure 2 as an example. If the result of the subtraction operation is greater than 0 , it indicates that the molecule concentration at the next moment is greater than the molecule concentration at the previous moment, and the counter is increased by one. Then, we continue to calculate the next difference value of two adjacent samples. At the end of the sampling process, if the value of the counter exceeds the threshold of counter Thre_counter, it indicates a significant rise during the sampling time. Then the signal is demodulated as a bit " 1 "; otherwise, the signal is demodulated as a bit " 0 ".

The IDA is very simple and has low complexity. During a single slot, each instruction of IDA is executed only once; the IDA in a single time slot is with a constant complexity of order, i.e., with the complexity of $\mathrm{O}(1)$. Considering that it is necessary to implement the IDA for multiple time slots, the complexity is added with time slot dimensionality, expressed by,

$$
T(n)=O(n) .
$$

The IDA can effectively reduce the BER, and significantly mitigate the influence of diffusion noise in the diffusion channel and ISI due to the accumulation of molecules around $\mathrm{Rx}$, especially for receiving " 0 " after a long sequence of " 1 ". In Fig.2, during the time between $T_{2}$ and $T_{2}+\tau$, although the absolute value of molecule concentration is very high, the descent is still able to be detected, which leads to the result demodulated as a bit " 0 ". Compared with other demodulation algorithms, the most significant advantage of the IDA is its property of eliminating the impact of ISI and versatility. Also, 


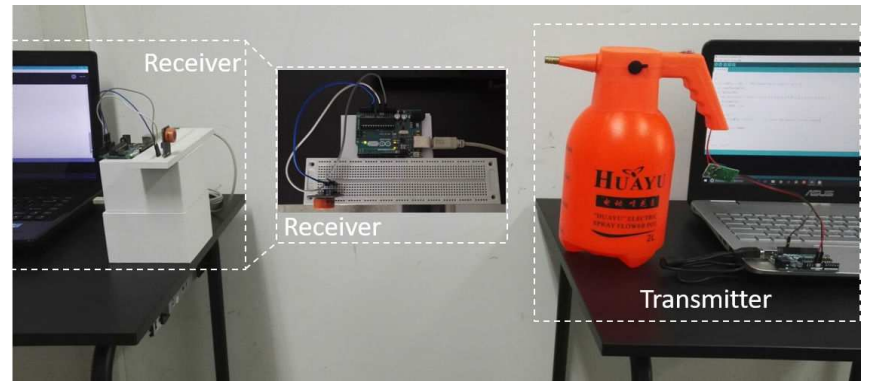

Fig. 3. The tabletop testbed with the MQ-3 metal-oxide sensor for molecular communication.

the IDA uses computationally simple operations, although its biological feasibility remains to be examined.

\section{EXPERIMENTAL SETUP}

In this section, we first introduce the tabletop testbed used for the experiments in this paper. Then, some fundamental experiments are performed on this platform to get some basic parameters, such as the transceiver distance $d$ and the length of each time slot $T$.

The first tabletop molecular communication platform designed to be inexpensive and simple is presented in [26], which is a powerful tool to facilitate the theoretical research. In this paper, the macroscale tabletop testbed used for experiments, which is similar to the first hardware platform except for the propagation channel without flow assisting, is shown in Fig. 3. The transmitter is composed of a spray for releasing alcohol molecules, an Arduino Uno microprocessor for controlling the on-off of the spray by a custom electrical switch board, and a computer to upload the controlling codes to the microprocessor. When an input is given to the computer, the information is converted into a binary sequence, which can be transmitted by controlling the spray precisely with OOK.

The signaling chemical used for transmission of information in this paper is isopropyl alcohol. When the spray releases these molecules, they propagate through the medium (i.e., air) based on diffusion and reach the receiver finally.

The receiver consists of an alcohol sensor and a microprocessor which reads the sensor data. Since isopropyl alcohol is used as the carrier of information, MQ-3 semiconducting metal oxide gas sensor, which can measure the concentration of different types of alcohol, is used for detecting molecule concentration around the receiver. The microprocessor at the receiver reads the sensor data by an analog to digital converter. The data then are sent to the computer through a serial port, which runs the proposed IDA and traditional demodulation algorithm. The results of these demodulation algorithms are displayed on the screen finally.

First, we present a series of impulse response of the overall system at different distances to choose the optimal value of $d$ relatively. To make sure there is a noticeable rise responding to the impulse in each time slot, then a sequence of " 1 " is sent continuously by $\mathrm{Tx}$ to get an apposite value of $T$. In addition, these experiments have been conducted in different directions by adjusting the angle from the central axis (i.e., the
TABLE II

EXPERIMENT PARAMETERS

\begin{tabular}{lccc}
\hline Parameter & Symbol & Value & Unit \\
\hline Time slot length & $T$ & $1-10$ & $\mathrm{~s}$ \\
Tx-Rx distance & $d$ & $60-140$ & $\mathrm{~cm}$ \\
Number of samples & $N \_$sample & 10 & $/$ \\
Threshold of counter & Thre_counter & 8 & $/$ \\
Fixed threshold & $C \_$max $/ 2$ & $0.6-1.2$ & $\mathrm{v}$ \\
Number of sent bits & N_bits & $1-10000$ & $/$ \\
\hline
\end{tabular}

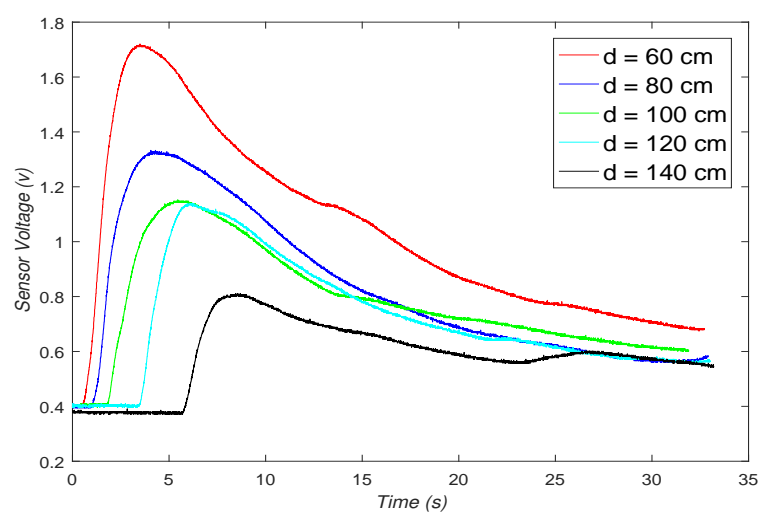

Fig. 4. The system response for different distances $d$ from Tx to Rx. $d$ varies from $60 \mathrm{~cm}$ to $140 \mathrm{~cm}$

suppositional line connecting the center of spray and sensor), while the experimental results are not affected by this factor. In this paper, we use the parameter values in Table 2 to conduct experiments. The length of each time slot $T$ varies from 2 to 10 s. And we set the transceiver distance $d$ as 60 to $140 \mathrm{~cm}$. The number of samples $N \_$sample in each time slot is set to 10 for simplicity, while the threshold of counter Thre_counter is 80 percent of $N_{-}$sample. Furthermore, we set the fixed threshold used in traditional demodulation algorithm as the half of maximum molecule concentration $C_{-} \max / 2$, which is represented by the voltage of sensor at $\mathrm{Rx}$ and varies from 0.6 to $1.2 \mathrm{v}$. And the number of sent bits at Tx varies from 1 to 10000 in different experiments. However, these values may not be optimal, which will be improved especially in future works.

Fig. 4 compares the system response for different separation distances from 60 to $140 \mathrm{~cm}$. As expected, the amplitude of the peak decreases and the delay before the peak increases as the separation distance $d$ increases. From the system response, it is evident that there is a significant decline from the peak voltage when the transceiver distance exceeds $120 \mathrm{~cm}$. And the sensor's resume time, i.e., the time it takes for the sensor to be used reliably again after a change in concentration, becomes much longer as $d$ increases. On the other hand, the time needed for the sensor to reach the peak from initial voltage will decrease as the parameter $d$ decreases, according to (9). Therefore, we set the value of distance $d$ from Tx to $\mathrm{Rx}$ as $80 \mathrm{~cm}$ in the following experiments.

Fig. 5 shows how the molecule concentration changes with time at the $\mathrm{Rx}$ when $\mathrm{Tx}$ sends a sequence of "111111111100...". The time slot length $T$ is 2,5 or 10 


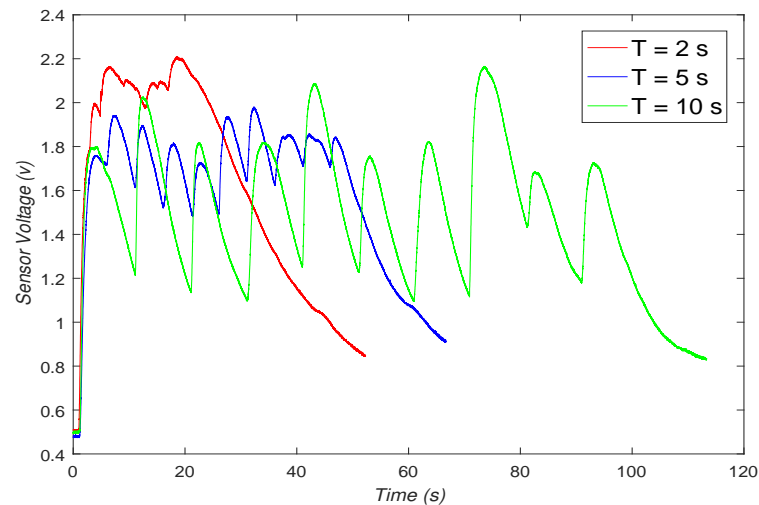

Fig. 5. Sensor voltages at Rx when Tx sends a sequence of "111111111100..." for various $T . d=80 \mathrm{~cm}$.

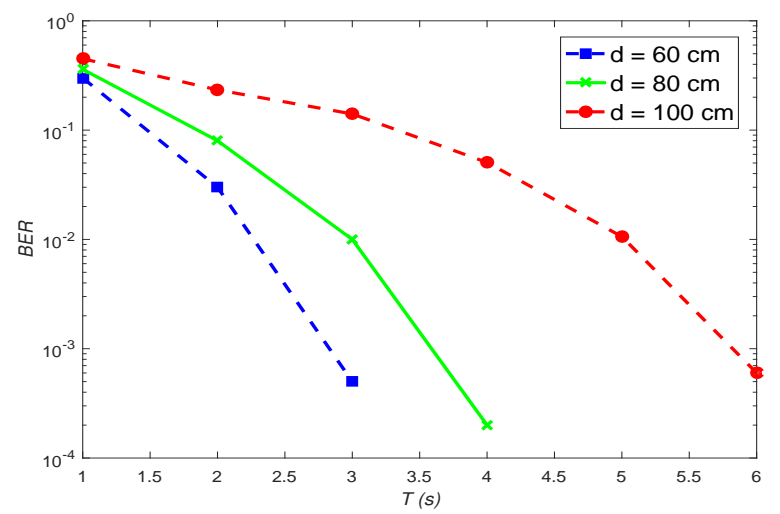

Fig. 6. BER under different $T$ with different $d$.

$\mathrm{s}$ respectively. The molecule concentration increases with oscillation because Tx always emits $M$ molecules at the beginning of each time slot to transmit a sequence of " 1 ". We note that the absolute value of molecule concentration at the $\mathrm{Rx}$ continues to increase, but the IDA would allow $\mathrm{Rx}$ to receive the sequence correctly. This is expected because there is always a rising edge at the beginning of each time slot, and $\mathrm{Rx}$ can demodulate the signal as long as it performs sampling in the interval where the molecule concentration rises. With the IDA, ISI could be avoided as long as the rising edge can be detected at the beginning of each time slot. In this paper, the time slot length $T$ is set as $5 \mathrm{~s}$.

\section{Vi. Performance Evaluation}

In this section, we evaluate the performance of the IDA proposed in this paper by the macroscopic molecular communication system using the set parameters previously. Then the IDA is compared with the common demodulation technique which is based on a fixed threshold in molecule concentration. And Tx transmits the information bit "0" or " 1 " randomly. Actually, we expect to the compare the performance with the latest ISI mitigation method, the ATV algorithm proposed in [19], while the ATV algorithm can't be implemented on this tabletop platform due to the differences in the used mathematical models. Finally, we transfer the test phrase "HELLO" from the transmitter to the receiver.

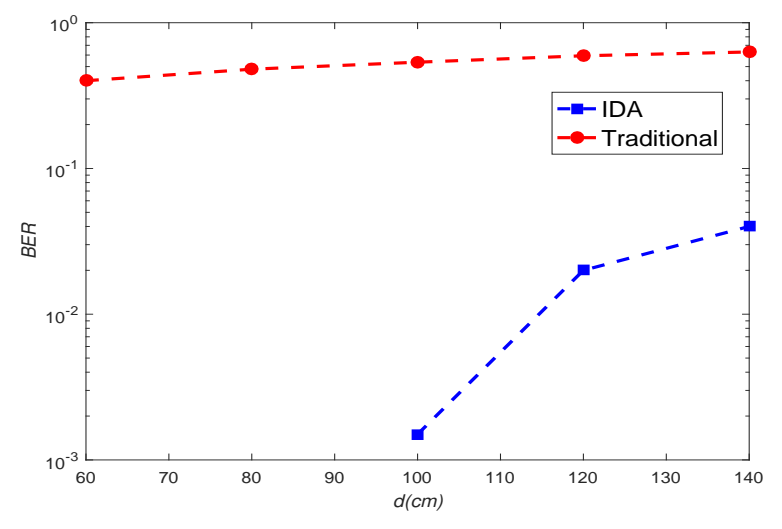

Fig. 7. BER under different $d$ with IDA or the traditional demodulation scheme. $T=5 \mathrm{~s}$.

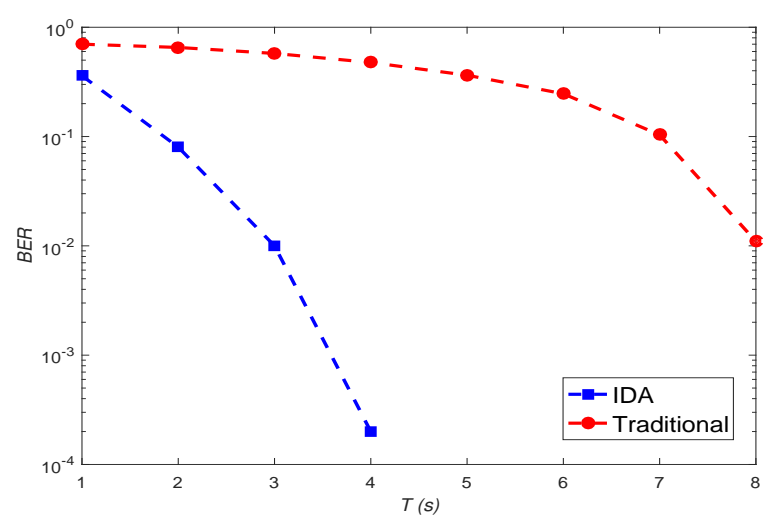

Fig. 8. BER under different $T$ with IDA or the traditional demodulation scheme. $d=80 \mathrm{~cm}$.

Fig. 6 shows the relationship between $B E R$ and $T$ at different transceiver distance $d$, which varies from 60 to 80 $\mathrm{cm}$. The performance of $B E R$ becomes better as the time slot $T$ increases, which weakens the effect of sensor's resume time. We can also see that larger the distance $d$ is, worse the $B E R$ performance is. These results are expected and can also be derived from Fig. 6, because an increase in $d$ directly results in a decrease in the number of molecules that arrive at Rx.

Fig. 7 compares the BER performance of IDA and that of the traditional demodulation technique which depends on a fixed concentration threshold (i.e., "Traditional" in the figure) as the transceiver distance varies from 60 to $140 \mathrm{~cm}$. In traditional demodulation technique, Rx compares the molecule concentration against the fixed molecule concentration threshold. The signal will be demodulated as a bit " 1 " if the molecule concentration obtained by $\mathrm{Rx}$ is above the threshold; otherwise, it will be received as a bit " 0 ". The performance of $B E R$ becomes worse as the Tx-Rx distance $d$ increases. As for the traditional demodulation algorithm depending on a fixed threshold, $B E R$ stabilizes at 0.5 according to the experiments, which is much worse than the performance with IDA. The reason might be that the time it takes for the sensor at $\mathrm{Rx}$ to resume to the initial state is very long, which is much greater than $5 \mathrm{~s}$ in the scenario while Tx transmits " 0 " or " 1 " randomly.

Fig. 8 shows the relationship between $B E R$ and $T$ with 


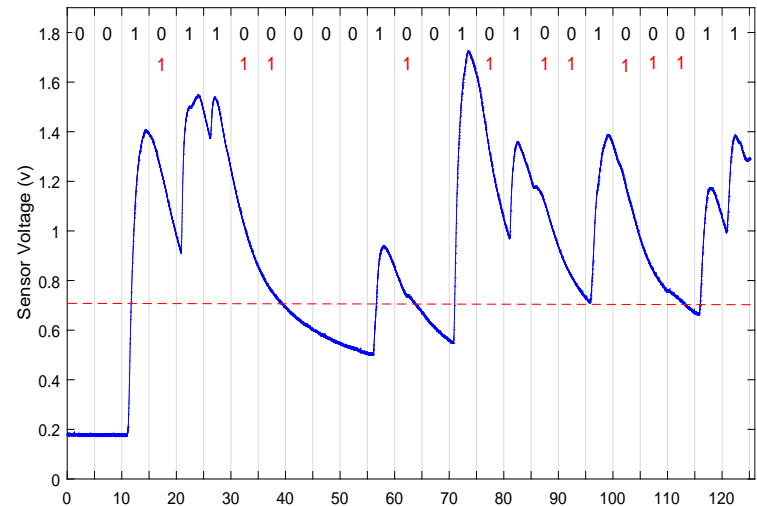

Fig. 9. Received signal when a 25-bit test sequence "00101 1000001001 0100100011 " is transmitted. $d=80 \mathrm{~cm}, T=5 \mathrm{~s}$.

IDA or the traditional demodulation scheme. Different from Fig. 7, the $B E R$ decreases as the length of time slot $T$ increases in Fig. 8. This is because the impact of ISI gradually decreases as $T$ increases, even that the impact of ISI will diminish when the time slot is large enough. These results demonstrate that BER using IDA is always much smaller than the case with the traditional demodulation method. IDA can significantly mitigate the impact of ISI in diffusion-based molecular communication and increase the information rate by shortening the length of each time slot.

Fig. 9 plots the sensor voltage reading during transmission session when a 25-bit test sequence "0010110000010010100100011 " is transmitted at the distance of $80 \mathrm{~cm}$ away. Solid black lines in vertical are used to distinguish each time slot, while the black digits corresponding to each time slot are the results demodulated by $\mathrm{Rx}$ with IDA. The dashed red line in horizontal presents the fixed threshold of traditional demodulation method. If the voltage is greater than the threshold, the signal is detected as a bit " 1 "; otherwise it is demodulated as a bit " 0 ". However, some bits that should be " 0 " may be demodulated to " 1 " using this scheme because of the effect of ISI, which are represented by the red numbers in this figure. In this paper, we use the International Telegraph Alphabet No. 2 (ITA2.0) standard to convert the text message to a binary sequence, where each letter is represented using five bits. As shown in Fig. 9, we are able to successfully transfer the test phrase "HELLO" from the transmitter to the receiver and avoid the interference caused by fluctuations in the curve, while the BER is $40 \%$ with the traditional demodulation algorithm.

\section{CONCLUSION}

In this paper, we investigate the reliability of concentrationencoded diffusion-based molecular communication. First, we present the basic system model consisting of a single transmitter, a single receiver and a diffusion-channel. We then design the IDA for a receiver to demodulate the information encoded in molecule concentration. The IDA aims to improve the BER performance by reducing the impact of ISI and other types of noise. Then some experiments are carried out using the tabletop molecular communication platform. Compared with the common demodulation technique depending on a fixed threshold in molecule concentration, the results of experiments show that the proposed IDA is capable of decreasing the BER in noisy molecular communication channels. Experimental results also indicate that the IDA can improve the reliability and the data rate for concentration-encoded diffusion-based molecular communication.

\section{REFERENCES}

[1] T. Nakano, M. J. Moore, F. Wei, A. V. Vasilakos, and J. Shuai, "Molecular communication and networking: Opportunities and challenges," IEEE Trans. Nanobiosci., vol. 11, pp. 135-148, Jun 2012.

[2] T. Nakano, A. W. Eckford, and T. Haraguchi, Molecular Communication. Cambridge University Press, Sep 2013.

[3] N. Farsad, H. B. Yilmaz, A. W. Eckford, C.-B. Chae, and W. Guo, "A Comprehensive Survey of Recent Advancements in Molecular Communication," IEEE Commun. Surveys Tuts., vol. 18, no. c, pp. 1-29, 2016.

[4] B. A. et al., Essential Cell Biology. New York, USA: Garland Science, 2009.

[5] T. Nakano, T. Suda, Y. Okaie, M. J. Moore, and A. V. Vasilakos, "Molecular communication among biological nanomachines: A layered architecture and research issues," IEEE Trans. Nanobiosci., vol. 13, no. 3, pp. 169-197, Sep 2014.

[6] H. Zhai, T. Nakano, A. V.Vasilakos, K. Yang, and Q. Liu, "Increase detection algorithm for concentration-encoded diffusion-based molecular communication," in Proc. ACM NanoCom, Washington DC, USA, Sep 2017.

[7] M. Pierobon and I. F. Akyildiz, "Information capacity of diffusion-based molecular communication in nanonetworks," in Proc. IEEE Int. Conf. Comput. Commun. (INFOCOM), Apr 2011, pp. 506-510.

[8] T. Nyberg, O. Inganäs, and H. Jerregärd, "Polymer hydrogel microelectrodes for neural communication," Biomedical Microdevices, vol. 4, no. 1, pp. 43-52, 2002.

[9] N. Farsad, A. W. Eckford, and S. Hiyama, "A markov chain channel model for active transport molecular communication," IEEE Trans. Signal Process., vol. 62, no. 9, pp. 2424-2436, May 2014.

[10] Y. Chahibi, I. F. Akyildiz, and S. O. Song, "Antibody-based molecular communication for targeted drug delivery systems," in Proc. 36th Annu. Int. Conf. IEEE Eng. Med. Biol. Soc. (EMBC), Aug 2014, pp. 57075710 .

[11] L. C. Cobo and I. F. Akyildiz, "Bacteria-based communication in nanonetworks," Nano Commun. Netw., vol. 1, no. 4, pp. 244-256, Dec 2010.

[12] M. U. Mahfuz, D. Makrakis, and H. T. Mouftah, "On the characterization of binary concentration-encoded molecular communication in nanonetworks," Nano Commun. Netw., vol. 1, no. 4, pp. 289-300, Dec 2010.

[13] —, "A comprehensive analysis of strength-based optimum signal detection in concentration-encoded molecular communication with spike transmission," IEEE Trans. Nanobiosci., vol. 14, no. 1, pp. 67-83, Jan 2015.

[14] — "A Comprehensive Study of Sampling-Based Optimum Signal Detection in Concentration-Encoded Molecular Communication," IEEE Trans. Nanobiosci., vol. 13, no. 3, pp. 208-222, Sep 2014.

[15] D. Kilinc and O. B. Akan, "Receiver design for molecular communication," IEEE J. Sel. Areas Commun., vol. 31, no. 12, pp. 705-714, Dec 2013.

[16] A. Noel, K. C. Cheung, and R. Schober, "Optimal receiver design for diffusive molecular communication with flow and additive noise," IEEE Trans. Nanobiosci., vol. 13, no. 3, pp. 350-362, Sep 2014.

[17] B. Li, M. Sun, S. Wang, W. Guo, and C. Zhao, "Local Convexity Inspired Low-Complexity Noncoherent Signal Detector for Nanoscale Molecular Communications," IEEE Trans. Commun., vol. 64, no. 5, pp. 2079-2091, Jan 2016.

[18] M. Damrath and P. A. Hoeher, "Low-Complexity Adaptive Threshold Detection for Molecular Communication," IEEE Trans. Nanobiosci., vol. 15, no. 3, pp. 200-208, Apr 2016.

[19] P. He, Y. Mao, Q. Liu, and K. Yang, "Improving reliability performance of diffusion-based molecular communication with adaptive threshold variation algorithm," International Journal of Communication Systems, vol. 29, no. 18, pp. 2669-2680, Dec 2016. 
[20] P. J. Shih, C. H. Lee, and P. C. Yeh, "Channel codes for mitigating intersymbol interference in diffusion-based molecular communications," in Proc. IEEE IEEE Global Commun. Conf. (GLOBECOM), Dec 2012, pp. 4228-4232.

[21] M. Pierobon and I. F. Akyildiz, "Intersymbol and co-channel interference in diffusion-based molecular communication," in Proc. Int. Conf. Commun.(ICC), Jun 2012, pp. 6126-6131.

[22] B. D. Unluturk, D. Malak, and O. B. Akan, "Rate-delay tradeoff with network coding in molecular nanonetworks," IEEE Trans. Nanotechnol., vol. 12, no. 2, pp. 120-128, Mar 2013.

[23] M. S. Leeson and M. D. Higgins, "Forward error correction for molecular communications," Nano Commun. Netw., vol. 3, no. 3, pp. 161-167, Sep 2012.

[24] C. T. Chou, "Impact of receiver reaction mechanisms on the performance of molecular communication networks," IEEE Trans. Nanotechnol., vol. 14 , no. 2, pp. 304-317, Mar 2015.

[25] T. Nakano and T. Suda, "Molecular Communication Using Dynamic Properties of Oscillating and Propagating Patterns in Concentration of Information Molecules," IEEE Trans. Commun., vol. 65, no. 8, pp. 33863398, Aug 2017.

[26] N. Farsad, W. Guo, and A. W. Eckford, "Tabletop molecular communication: Text messages through chemical signals," PLOS ONE, vol. 8, no. 12, Dec 2013.

[27] N. R. Kim, N. Farsad, C. B. Chae, and A. W. Eckford, "A realistic channel model for molecular communication with imperfect receivers," in Proc. Int. Conf. Commun., Sydney, NSW, Australia, Jun 2014, pp. 3987-3992.

[28] N. Farsad, N.-R. Kim, A. W. Eckford, and C.-B. Chae, "Channel and Noise Models for Nonlinear Molecular Communication Systems," IEEE J. Sel. Areas Commun., vol. 32, no. 12, pp. 2392-2401, Dec 2014.

[29] N.-R. Kim, N. Farsad, C.-B. Chae, and A. W. Eckford, "A universal channel model for molecular communication systems with metal-oxide detectors," Proc. Int. Conf. Commun., pp. 1054-1059, Jun 2015.

[30] C. Lee, B. Koo, N. R. Kim, B. Yilmaz, N. Farsad, A. Eckford, and C. B. Chae, "Molecular MIMO communication link," in Proc. IEEE Int. Conf. Comput. Commun. (INFOCOM), Hong Kong, Apr 2015, pp. 13-14.

[31] C. Lee, B. Koo, N.-R. Kim, H. B. Yilmaz, N. Farsard, A. Eckford, and C.-B. Chae, "Molecular MIMO with drift," in Proc. ACM MobiCom, New York, USA, 2015, pp. 201-203.

[32] M. S. Kuran, H. B. Yilmaz, T. Tugcu, and B. Özerman, "Energy model for communication via diffusion in nanonetworks," Nano Commun. Netw., vol. 1, no. 2, pp. 86-95, Jun 2010.

[33] M. S. Kuran, H. B. Yilmaz, T. Tugcu, and I. F. Akyildiz, "Modulation techniques for communication via diffusion in nanonetworks," Proc. Int. Conf. Commun., pp. 1-5, Jun 2011.

[34] H. Arjmandi, A. Gohari, M. N. Kenari, and F. Bateni, "Diffusionbased nanonetworking: A new modulation technique and performance analysis," IEEE Commun. Lett., vol. 17, no. 4, pp. 645-648, Apr 2013.

[35] N. R. Kim and C. B. Chae, "Novel modulation techniques using isomers as messenger molecules for molecular communication via diffusion," in Proc. Int. Conf. Commun., Jun 2012, pp. 6146-6150.

[36] M. Pierobon and I. F. Akyildiz, "Diffusion-based noise analysis for molecular communication in nanonetworks," IEEE Trans. Signal Process., vol. 59, no. 6, pp. 2532-2547, Jun 2011.

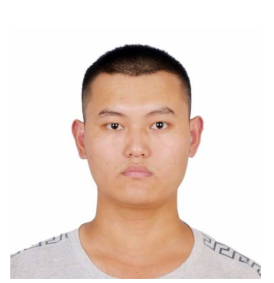

Haoyang Zhai received the B. Eng. degree in network engineering from School of Communication and Information Engineering, University of Electronic Science and Technology of China, in 2016, where he is currently pursuing the M.Eng. degree in electronic and communication engineering. His research interest lies in molecular communication.

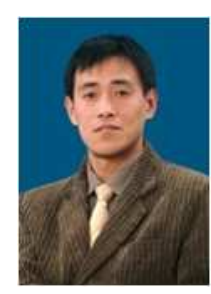

Qiang Liu received his B. Eng.(1996), M. Eng.(2000) and Ph.D(2012) from University of Electronic Science and Technology of China (UESTC). After graduating from ME study in 2000, he has worked with School of Communication and Information Engineering, UESTC, China, and is an associate professor now. His research focuses on wireless sensor networks, Internet of Things, broadband wireless networks and molecular communication. He is the member of IEEE.

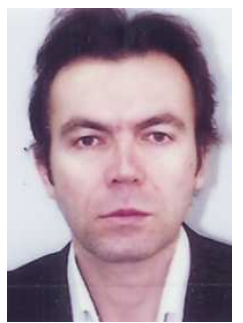

Athanasios V. Vasilakos is recently Professor with the Lulea University of Technology, Sweden. He served or is serving as an Editor for many technical journals, such as the IEEE Transactions on Network and Service Management; IEEE Transactions on Services Computing; IEEE Transactions on Cloud Computing, IEEE Transactions on Information Forensics and Security, IEEE Transactions on Cybernetics; IEEE Transactions on Nanobioscience; IEEE Transactions on Information Technology in Biomedicine; ACM Transactions on Autonomous and Adaptive Systems; the IEEE Journal on Selected Areas in Communications.

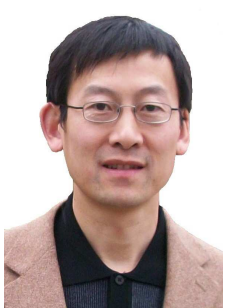

Kun Yang received his Ph.D. from the Department of Electronic and Electrical Engineering of University College London (UCL), UK, and M.Sc. and B.Sc. from the Computer Science Department of Jilin University, China. He is currently a Chair Professor in the School of Computer Science and Electronic Engineering, University of Essex, leading the Network Convergence Laboratory (NCL), UK. He is also an affiliated professor at UESTC, China. His main research interests include wireless networks and communications, data and energy integrated networks, computation-communication cooperation. He serves on the editorial boards of both IEEE and non-IEEE journals. He is a Senior Member of IEEE and a Fellow of IET. 